\title{
Erdschlüsse bei Mittelspannungskabeln und die elektrische Beeinflussung im Bereich von Swimmingpools
}

\author{
Katrin FriedI (D) - Reinhard Hirtler · Lothar Fickert · René Braunstein · Robert Schürhuber
}

Eingegangen: 3. Oktober 2021 / Angenommen: 30. November 2021 / Online publiziert: 16. Februar 2022

(c) Der/die Autor(en) 2022

\begin{abstract}
Zusammenfassung Bei Erdschlüssen an Mittelspannungskabeln können in unmittelbarer Umgebung der Fehlerstelle durch den Eintritt von Strömen in das Erdreich Potentialanhebungen und somit Schrittund Berührungsspannungen auftreten. Diese müssen hinsichtlich des Risikos einer Personengefährdung betrachtet werden. Eine besondere Situation entsteht dabei, wenn der Fehler im Nahbereich eines Swimmingpools auftritt, da sich hier Personen barfuß aufhalten können. Die dabei kleineren Übergangswiderstände zum Erdreich als bei Personen mit Schuhwerk führen zu größeren Körperströmen. Diese Situation wird unter Berücksichtigung der einschlägigen Normen auf ihr Gefährdungspotential beurteilt. Im Zentrum dieses Fachbeitrages steht die Frage, unter welchen Bedingungen es bei der Annäherung von Swimmingpool und erdverlegtem Kabel bei einem Erdschluss in kompensierten Mittelspannungsnetzen $(20 \mathrm{kV}$ und $30 \mathrm{kV})$ zu einer Personengefährdung kommen kann und welche Maßnahmen von den Betreibern dieser Kabel erforderlichenfalls $\mathrm{zu}$ treffen sind.

Zwei unterschiedliche Fehlerszenarien werden im Detail analysiert: ein Kabelfehler in der Nähe eines Swimmingpools sowie ein Fehler im Bereich eines Kabelaufführungsmastes oder in einer Ortsnetzstation mit Potentialverschleppung über den Kabelbegleiterder.
\end{abstract}

K. Friedl, OVE (ه) $\cdot$ L. Fickert, OVE $\cdot$ R. Schürhuber, OVE Institut für Elektrische Anlagen und Netze, Technische Universität Graz, Inffeldgasse 18, 8010 Graz, Österreich katrin.friedl@tugraz.at

R. Hirtler, OVE

Wien, Österreich

R. Braunstein, OVE

Energie Steiermark Technik GmbH, Graz, Österreich
Die wesentlichen Einflussparameter werden hinsichtlich der Auswirkungen auf den Fehlerstrom sowie auf Schritt- und Berührungsspannungen untersucht. Aus den Untersuchungen geht hervor, dass bei den meisten betrachteten Fehlerszenarien keine Gefährdung für Personen hinsichtlich Schritt- und Berührungsspannungen zu erwarten ist. Einzig der Fehlerfall mit Potentialverschleppung über den Kabelbegleiterder in Gebieten mit spezifischen Bodenwiderständen von größer als $1000 \Omega \mathrm{m}$ muss im Anlassfall geprüft werden.

Schlüsselwörter Mittelspannungskabel .

Erdungsanlagen · Erdschlüsse · Kabelfehler · Schutz vor elektrischem Schlag · Berührungsspannungen · Kabelschirme $\cdot$ Barfußbereich

\section{Earth faults in medium voltage cables and the electrical interference in the vicinity of swimming pools}

\begin{abstract}
In the case of earth faults in medium voltage cables, potential rises and thus step and touch voltages can occur in the immediate vicinity of the fault location due to the injection of currents into the ground. These have to be examined with regard to personal safety. A special situation arises when a fault occurs in the vicinity of a swimming pool since people could be barefoot in this area. There are smaller contact resistances to the ground for barefoot people than for people with footwear leading to larger body currents. This situation is assessed in terms of its hazard potential, taking the relevant standards into account. This paper focuses on the following question: Under which conditions can the proximity of a swimming pool to a buried cable result in a hazard to humans in the event of an earth fault in compensated medium voltage networks ( $20 \mathrm{kV}$ and $30 \mathrm{kV})$, and, if necessary,
\end{abstract}


what measures should be taken by the operators of these cables?

Two different fault scenarios are analyzed in detail: a cable fault in the vicinity of a swimming pool and a fault in the area of a cable transition tower with transferred potential via the cable accompanying earth electrode.

The main parameters influencing the fault current are investigated with respect to the effects on step and touch voltages.

The investigations show that in most of the fault scenarios considered, no hazard is to be expected for humans with regard to step and touch voltages. Only the special fault scenario with transferred potential via cable accompanying earth electrodes in areas with soil resistivities higher than $1000 \Omega \mathrm{m}$ have to be investigated in more detail.

Keywords Medium voltage cables · Earthing arrangements - Earth faults - Cable faults - Protection against electric shock - Touch voltages - Cable shields · Barefoot area

\section{Einleitung und Methodik}

Durch die steigende Beliebtheit von Swimmingpools und dem gleichzeitig steigenden Verkabelungsgrad in urbanen und suburbanen Gebieten kommt es vermehrt zu Anfragen hinsichtlich etwaiger Personengefährdung durch Annäherungen zwischen Mittelspannungskabeln und Swimmingpools. Im Bereich von Swimmingpools halten sich Personen barfüßig auf. Dies führt dazu, dass verringerte Zusatzwiderstände als bei Personen mit Schuhwerk bei der Beurteilung von Schritt- und Berührungsspannungen berücksichtigt werden müssen.

Mittelspannungskabel können aus Alterungsgründen, durch Beschädigung der Isolierung im Zuge der Verlegung oder durch äußere Einflüsse (z.B. Grabungsarbeiten, Schalthandlungen, Blitzschläge) ihre Isolationsfestigkeit verlieren. Dies führt in weiterer Folge zu Isolationsfehlern, meist zu einpoligen Erdschlüssen. Bei solchen Erdschlüssen kann ein Teil des Fehlerstroms $I_{\mathrm{F}}$ an der Fehlerstelle in das umgebende Erdreich treten. Der Stromanteil über Erde, der Erdungsstrom $I_{\mathrm{E}}$, ist für eine Anhebung des Potentials an der Erdoberfläche im Bereich des erdverlegten Kabels und somit für eine etwaige Gefährdung von Personen durch Schritt- und Berührungsspannungen verantwortlich. Tritt ein Erdschluss in einer Ortsnetzstation (Transformatorstation) oder bei einem Kabelaufführungsmast auf, so fließen in den daran angeschlossenen Kabelbegleiterdern Erdungsströme. Diese führen ebenso zu Potentialanhebungen entlang der Kabeltrasse und damit auch im Bereich von Swimmingpools.

Mittelspannungsnetze werden im D-A-CH-Raum überwiegend mit Erdschlusskompensation betrieben. Im Vergleich zu Netzen mit niederohmig geerde- tem Sternpunkt treten bei erdschlusskompensierten Netzen vergleichsweise kleine Erdschlussströme auf. Da gemäß ÖVE-B 1/1976 [1] die Notwendigkeit für weiterführende Untersuchungen hinsichtlich der induktiven Beeinflussung von Fernmeldeanlagen bei Erdschlussrestströmen bis zu $60 \mathrm{~A}$ in $20-\mathrm{kV}$-Netzen bzw. bei $67 \mathrm{~A}$ in 30-kV-Netzen mit Erdschlusskompensation entfallen darf, wurden diese Stromwerte in der Betriebspraxis bisher häufig als Orientierungswert für Erdschlussüberlegungen herangezogen. Betriebsinterne Festlegungen in technischen Ausführungsbestimmungen für die Auslegung der Erdungsanlagen von Ortsnetzstationen berücksichtigen meist diesen Orientierungswert. Daher werden auch die hier beschriebenen Untersuchungen auf Basis dieser Stromwerte durchgeführt.

Erdschlüsse im Bereich von Mittelspannungskabeln werden in Netzen mit Erdschlusskompensation nach erfolgreicher Fehlerortung so rasch wie möglich behoben. Entsprechend der jeweiligen Betriebsphilosophie kann das erdschlusskompensierte Netz aufgrund des vergleichsweise kleinen Fehlerstromes jedoch auch längere Zeit mit anstehendem Erdschluss weiterbetrieben werden. Dies bedeutet bei der Betrachtung der Personengefährdung, dass in diesem Fall für die Bestimmung der höchstens zulässigen Berührungsspannung $U_{\mathrm{Tp}}$ Fehlerdauern $t_{\mathrm{F}}$ von größer als 10 s herangezogen werden müssen. Gemäß ÖVE/ÖNORM EN 50522:2011 [2] ergibt sich damit als Grenzwert der zulässigen Berührungsspannung $U_{\mathrm{Tp}}=80 \mathrm{~V}$.

Die im Folgenden zu untersuchenden Fragestellungen, welche für Netzbetreiber und Anrainer von Interesse sind, lauten daher:

- Wie groß ist das Risiko einer Gefährdung von Personen im Annäherungsbereich von Mittelspannungskabel und Swimmingpools im Erdschlussfall?

- Welche Maßnahmen müssen bei Mittelspannungskabeln ergriffen werden, um ein etwaiges Risiko auf ein vertretbares Maß zu reduzieren?

In diesem Fachbeitrag werden zwei Fehlerszenarien untersucht, welche zu einer Personengefährdung führen könnten:

- Erdschlüsse bei Kabelfehler in unmittelbarer Umgebung eines Swimmingpools

- Erdschlüsse in einer nahegelegenen Ortsnetzstation oder an einem Kabelaufführungsmast und die dadurch verursachten Potentialverschleppungen über mitverlegte Kabelbegleiterder im Bereich von Swimmingpools

In Abschn. 2 werden die im Zusammenhang mit den Fehlerszenarien und der Erdung bestehenden Begriffe beschrieben. Abschn. 3 behandelt das Fehlerszenario von Kabelfehlern und Abschn. 4 die Auswirkung der Potentialverschleppung über Kabelbegleiterder. Erkenntnisse werden in Abschn. 5 zusammengefasst. 


\section{Wesentliche Begriffe bezüglich Erdung}

Die folgenden Begriffe sind in Anlehnung an [2] für die oben angeführten Fragestellungen wesentlich.

Für eine Anhebung des Potentials an der Erdoberfläche und somit für eine etwaige Gefährdung durch Schritt- oder Berührungsspannungen ist der Erdungsstrom $\underline{I}_{\mathrm{E}}$ verantwortlich. Dies ist jener Teil des Erdfehlerstromes $\underline{I}_{\mathrm{F}}$, der an der Fehlerstelle in das Erdreich fließt. Ein Erder ist nach [2] ein „leitfähiges Teil, das [...] in elektrischem Kontakt mit Erde steht". Bei beschädigtem Kabelmantel und -schirm kann die blanke Fehlerstelle am Kabel wie ein Erder betrachtet werden.

Bei der Potentialanhebung im Fehlerfall wird als Bezug ein weit entfernter Punkt - die "ferne Erde“ oder Bezugserde - herangezogen. Die Erdungsspannung $\underline{U}_{\mathrm{E}}$ ist in weiterer Folge die Spannung zwischen dem Erder und der Bezugserde. Berührungsspannungen $U_{\mathrm{T}}$ sind Spannungsabfälle, die an Personen beim Berühren von leitfähigen Teilen auftreten; Schrittspannungen $U_{\mathrm{s}}$ sind Spannungsabfälle bei einem Stromweg von einem Fuß zum andern. Die Schrittweite $S$ ist dabei mit $1 \mathrm{~m}$ festgelegt.

Die Erdungsimpedanz $\underline{Z}_{E}$ einer Erdungsanlage ergibt sich aus dem Verhältnis von Erdungsspannung $\underline{U}_{E}$ und Erdungsstrom $\underline{I}_{\mathrm{E}}$. Der Ausbreitungswiderstand $R_{\mathrm{E}}$ bezeichnet den Widerstand zwischen einem örtlich begrenzten Erder und Bezugserde.

Gefährlich für Personen ist insbesondere ein Stromfluss über das Herz, welcher groß genug ist, um Herzkammerflimmern hervorzurufen. Auf der Basis von 5-\%-Schwellwerten für Körperströme $I_{B}$ gemäß [3] sindmittels einer Gewichtung für verschiedene Strompfade durch den Körper in [2] zulässige Berührungsspannungen $U_{\text {Tр }}$ in Abhängigkeit der Fehlerdauer $t_{\mathrm{f}}$ festgelegt. Die höchste zulässige Berührungsspannung $U_{\text {Tp }}$ ergibt sich nach [2] für Fehlerdauern $t_{\mathrm{f}}>1 \mathrm{~s}$ zu $80 \mathrm{~V}$. Zulässige Schrittspannungen sind in [2] nicht angegeben, können aber mit dem dort beschriebenen Berechnungsverfahren ermittelt werden. Ein Strompfad Fuß zu Fuß ist für das Herz wesentlich unkritischer als die anderen Strompfade wie z.B. Hand zu Fuß. Daher können für Schrittspannungen wesentlich höhere Grenzen als für Berührungsspannungen zugelassen werden.

Nach [2] kann mit Zusatzwiderständen für z.B. Schuhwerk oder dem Ausbreitungswiderstand des Standortes auf eine höchste zulässige Leerlauf-Berührungsspannung $U_{\mathrm{vTp}}$ umgerechnet werden. Die Leerlauf-Berührungsspannung $U_{\mathrm{VT}}$ ist die Spannungsdifferenz zwischen zwei Punkten, an denen eine Berührung stattfinden kann, jedoch ohne tatsächlich Berührung, also ohne Stromfluss über den menschlichen Körper. Sie kann direkt aus dem Verlauf des an der Erdoberfläche gemessenen oder berechneten Potentials $\varphi$ ermittelt werden. In Abb. 1 sind für das Beispiel eines einfachen Erders E, der an einen metallenen Steher angeschlossen ist, im Verlauf des Oberflächenpotentials $\varphi$ die Leerlauf-Berührungs-

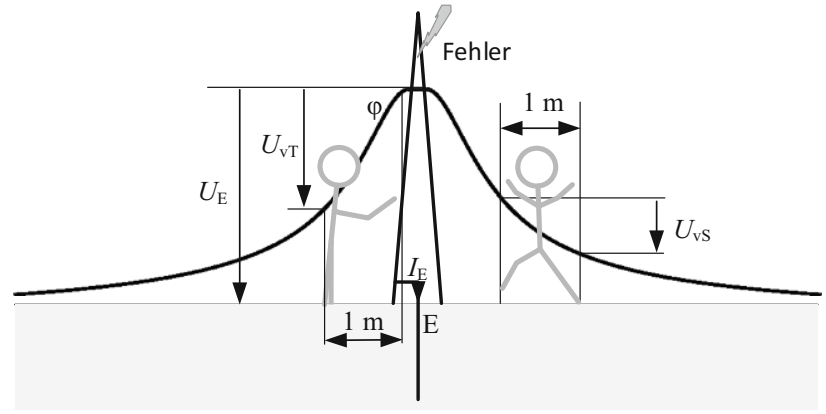

Abb. 1 Skizze für den Verlauf des Oberflächenpotentials $\varphi$ eines Erders $\mathrm{E}\left(\underline{U}_{\mathrm{E}}\right.$ Erdungsspannung, $U_{\mathrm{vT}}$ Leerlauf-Berührungsspannung, $U_{\mathrm{vS}}$ Leerlauf-Schrittspannung, $\underline{I}_{\mathrm{E}}$ Erdungsstrom)

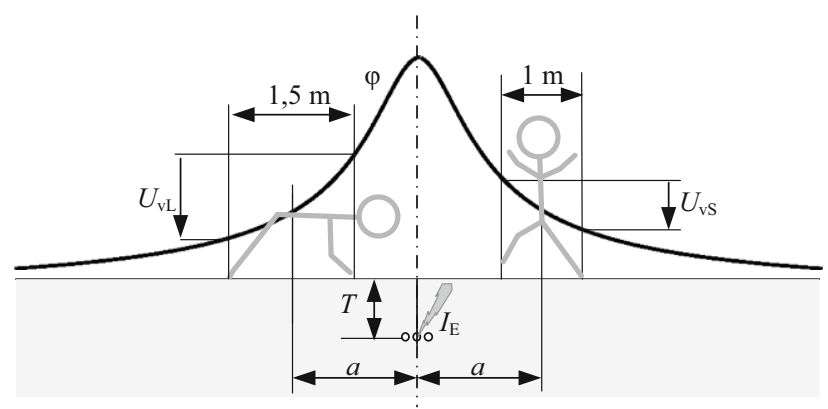

Abb. 2 Skizze für den Verlauf des Oberflächenpotentials $\varphi$ bei einem Kabelfehler $\left(U_{\mathrm{vL}}\right.$ Leerlauf-Liegestützspannung bzw. Leerlauf-Berührungsspannung einer liegenden Person, $U_{\mathrm{vs}}$ Leerlauf-Schrittspannung, $\underline{I}_{\mathrm{E}}$ Erdungsstrom, a horizontaler Abstand vom Fehler, $T$ Verlegetiefe des Kabels)

spannung $U_{\mathrm{vT}}$ und der Leerlauf-Schrittspannung $U_{\mathrm{vS}}$ skizziert.

In den betrachteten Fehlerszenarien im Bereich eines Swimmingpools ist eine Berührung der Erder wie in Abb. 1 dargestellt nicht möglich, da die Fehlerstelle bzw. der Kabelbegleiterder im Erdreich liegt. Jedoch ist ein Berührungsszenario einer Person etwa in Liegestützstellung nach Abb. 2 möglich. Nach ÖVEEH 41/1987 [4] war deshalb speziell für Campingplätze und Freibäder zu überprüfen, dass „die Spannung zwischen zwei beliebigen, 1,5m voneinander entfernten Punkten“ die zulässige Berührungsspannung nicht überschreitet. Im Folgenden wird daher zusätzlich zur Leerlauf-Schrittspannung $U_{\mathrm{vs}}$ die LeerlaufBerührungsspannung einer liegenden Person - im Folgenden als Leerlauf-Liegestützspannung $U_{\mathrm{vL}}$ bezeichnet - nach Abb. 2 betrachtet.

Die zulässigen Werte für die Leerlauf-Liegestützspannung werden nach [2, Anhang A] berechnet.

Für eine zulässige Liegestützspannung $U_{\text {Lp }}$ bzw. die zulässige Leerlauf-Liegestützspannung $U_{\text {vLp }}$ ergeben sich demnach mit Gl. 1 bzw. Gl. 2:

$$
U_{\mathrm{Lp}}=I_{B}\left(t_{\mathrm{f}}\right) \cdot \frac{1}{H F} \cdot Z_{\mathrm{T}}\left(U_{\mathrm{T}}\right) \cdot B F
$$




$$
U_{\mathrm{vLp}}=I_{\mathrm{B}}\left(t_{\mathrm{f}}\right) \cdot \frac{1}{H F}\left(Z_{\mathrm{T}}\left(U_{\mathrm{T}}\right) \cdot B F+R_{\mathrm{H}}+R_{\mathrm{F}}\right)
$$

mit:

$I_{\mathrm{B}}\left(t_{\mathrm{f}}\right) \quad$ Körperstrom für Kurve $\mathrm{c}_{2}$ aus [3], für $t_{\mathrm{f}}>10 \mathrm{~s}$ ergibt sich $I_{\mathrm{B}}=50 \mathrm{~mA}$

HF Herzstromfaktor aus [3], für den Strompfad beide Hände - beide Füße ergibt sich $H F=$ 1,0

$Z_{\mathrm{T}}\left(U_{\mathrm{T}}\right) \quad$ Körperimpedanz aus [3, Tab. 1], muss wegen der Abhängigkeit von $U_{\mathrm{T}}$ iterativ bestimmt werden

BF Korrekturfaktor für die Körperimpedanz; für den Strompfad beide Hände - beide Füße ergibt sich $B F=0,5$

$R_{\mathrm{H}}, R_{\mathrm{F}} \quad$ Zusatzwiderstände für Hand und Fuß, barfüßig für die Liegestützposition gegeben durch die Standortwiderstände von Händen und Füßen; analog nach [2, Anhang B] ergibt sich z. B. $R_{\mathrm{F}}=R_{\mathrm{H}}=1,5 \mathrm{~m}^{-1} \rho_{\mathrm{E}}$

Damit resultiert eine zulässige Liegestützspannung von $U_{\text {Lp }}$ von $58 \mathrm{~V}$ und eine zulässige Leerlauf-Liegestützspannung $U_{\mathrm{vLp}}$ von $73 \mathrm{~V}$ bei $\rho_{\mathrm{E}}=100 \Omega \mathrm{m}$ bzW. $208 \mathrm{~V}$ bei $\rho_{\mathrm{E}}=1000 \Omega \mathrm{m}$.

\section{Erdschlüsse bei Kabelfehlern}

In diesem Abschnitt werden die Modellierung, die Berechnung und die Ergebnisse für das Fehlerszenario Erdschluss bei einem Kabelfehler beschrieben. Ausgehend vom Ausbreitungswiderstand der Fehlerstelle in Abschn. 3.1 wird die Ermittlung der wirksamen Fehlerimpedanz in Abschn. 3.2 zuerst ohne Berücksichtigung der Kabelschirme und anschließend in Abschn. 3.3 mit Berücksichtigung der Schirme beschrieben. Ziel ist dabei das Bestimmen des Erdungsstromes $I_{\mathrm{E}}$ an der Fehlerstelle in Abhängigkeit von verschiedenen Parametern. Mit dem Erdungsstrom wird in Abschn. 3.4 der Potentialverlauf an der Erdoberfläche und daraus die Leerlauf-Schritt- und die Leerlauf-Liegestützspannung im Bereich des Kabel-

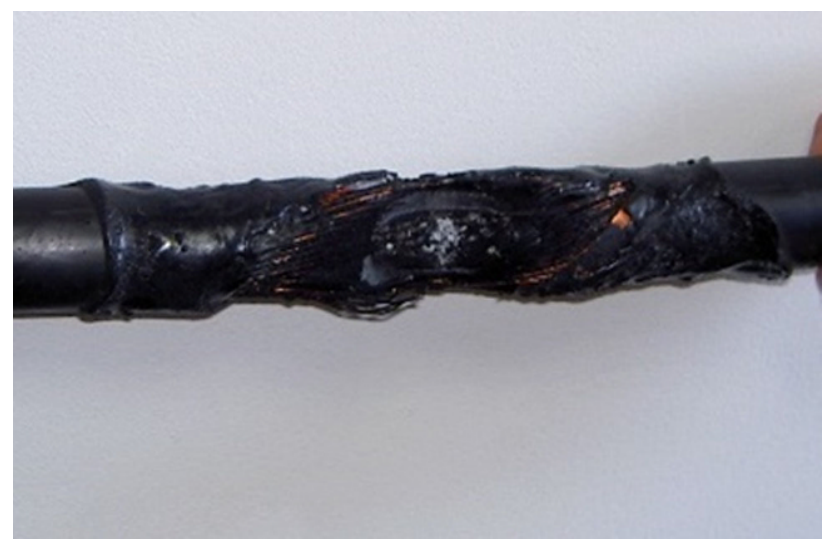

Abb. 3 Beispiel einer Fehlerstelle eines Mittelspannungskabels. (Quelle: eigenes Foto) fehlers ermittelt. In Abschn. 3.5 werden einige ausgewählte Parametervariationen hinsichtlich der Schrittund Berührungsspannungen bewertet.

\subsection{Modellierung der Fehlerstelle}

Unter der Annahme, dass sowohl der Kabelmantel als auch der Kabelschirm beschädigt sind, wird die blanke Stelle des Kabels an der Fehlerstelle wie ein Erder modelliert.

An der Fehlerstelle des Kabels (siehe Abb. 3) kann durch den entstehenden Lichtbogen ein Teil der Isolierung und des Schirmes sowie des Kabelmantels weggebrannt werden. Der Innenleiter liegt dann an dieser Stelle frei. Diese freiliegende metallene Fläche wirkt nun wie ein im Erdreich eingebetteter Erder. Für die Bestimmung des Ausbreitungswiderstandes $R_{\mathrm{E}, \mathrm{F}}$ wird die Fehlerstelle als Kreisplattenerder modelliert [5]:

$$
R_{\mathrm{E}, \mathrm{F}}=\frac{\rho_{\mathrm{E}}}{4 \sqrt{A / \pi}}
$$

mit:

$R_{\mathrm{E}, \mathrm{F}} \quad$ Ausbreitungswiderstand der Fehlerstelle

$\varrho_{\mathrm{E}} \quad$ spezifischer Widerstand des umgebenden Erdreichs

$A \quad$ Fläche der Fehlerstelle

Durch die Auswertung mehrerer Fehlerstellen bei Mittelspannungskabeln konnte für die blanken Stellen eine Fläche von höchstens etwa $10 \mathrm{~cm}^{2}$ bestimmt werden.

Eine genauere Modellierung der Fehlerstelle führt wegen der Unsicherheit bei wesentlichen Parametern - wie Größe der Fehlerstelle und spezifischer Bodenwiderstand des umgebenden Erdreichs - in der Praxis nicht zu genaueren Ergebnissen.

Für die als Kreisplattenerder modellierte Fehlerstelle ergibt sich daher beispielsweise bei einem spezifischen Bodenwiderstand von $\rho_{E}=100 \Omega \mathrm{m}$ ein Ausbreitungswiderstand $R_{\mathrm{E}, \mathrm{F}}$ von $1,4 \mathrm{k} \Omega$.

\subsection{Modellierung des Netzes}

Bei der Ermittlung von Potentialverläufen bei Erdfehlern wird häufig von eingeprägten Strömen ausgegangen. Hier wird dafür der höchste zu berücksichtigende Erdschlussreststrom $I_{\text {Fmax }}$ an der Fehlerstelle betrachtet. Nach Abschn. 1 wird dieser bei einem mit Erdschlusskompensation betriebenen 30-kV-Netz mit $67 \mathrm{~A}$, bei $20-\mathrm{kV}-$ Netzen mit $60 \mathrm{~A}$ angenommen.

Bei dem in Abschn. 3.1 bestimmten Ausbreitungswiderstand der Fehlerstelle $R_{\mathrm{E}, \mathrm{F}}$ von $1,4 \mathrm{k} \Omega$, würde dabei bei einem eingeprägten Fehlerstrom $I_{\mathrm{F}}$ von $67 \mathrm{~A}$ rechnerisch eine Erdungsspannung $U_{\mathrm{E}}$ (Spannung von der Fehlerstelle zur fernen Erde) von $93 \mathrm{kV}$ auftreten. Diese hohe Spannung kann jedoch bei einer Netznennspannung von $30 \mathrm{kV}$ weder stationär noch transient auftreten. Technisch korrekt wird das Netz in wei- 


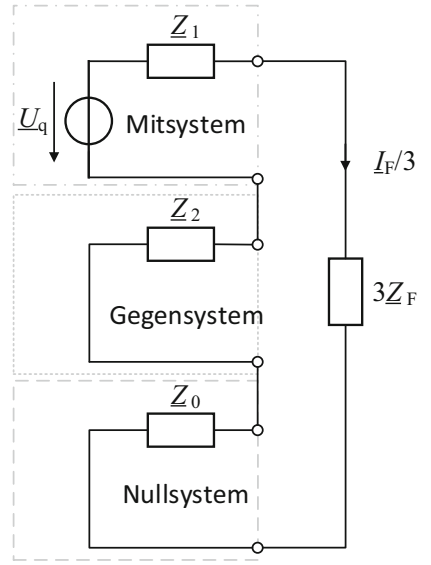

Abb. 4 Vollständiges Ersatzschaltbild in symmetrischen Komponenten im einpoligen Fehlerfall

terer Folge anstatt mit einer eingeprägten Stromquelle als Spannungsquelle mit Ersatzimpedanzen in symmetrischen Komponenten modelliert.

In symmetrischen Komponenten sind die Komponentensysteme bei einem einpoligen Fehler in Reihe zu schalten; es ergibt sich die in Abb. 4 dargestellte Ersatzschaltung. Dabei ist:

$\underline{U}_{\mathrm{q}} \quad$ Quellenspannung $\left(U_{\mathrm{n}} / \sqrt{3}\right)$

$\underline{Z}_{1}^{\mathrm{q}}, \underline{Z}_{2}, \underline{Z}_{0}$ Ersatzimpedanzen des Netzes von der Fehlerstelle aus betrachtet

$\underline{Z}_{\mathrm{F}} \quad$ Fehlerimpedanz

$\underline{Z}_{1}$ und $\underline{Z}_{2}$ sind in heute typischen kompensierten Netzen um den Faktor 20-200 kleiner als $\underline{Z}_{0}$ und können daher vernachlässigt werden. Damit ergibt sich das vereinfachte Ersatzschaltbild nach Abb. 5.

Die Resistanz der Ersatzimpedanz im Nullsystem $\underline{Z}_{0}$ kann hier ebenfalls vernachlässigt werden. Im typischerweise überkompensiert betriebenen Netz ergibt sich $\underline{Z}_{0}=\mathrm{j} X_{0}$. Die minimale Nullreaktanz $X_{0}$ ergibt sich aus:

$$
X_{0}=\frac{3 U_{\mathrm{q}}}{I_{\mathrm{F}, \max }}
$$

mit:

$X_{0} \quad$ Ersatzreaktanz des Netzes im Nullsystem von der Fehlerstelle aus gesehen

$U_{\mathrm{q}} \quad$ Quellspannung (höchste Netzspannung $U_{\mathrm{n}} / \sqrt{3}$ )

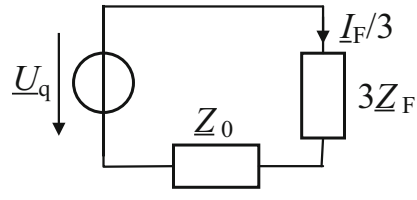

Abb. 5 Vereinfachtes Ersatzschaltbild im Komponentensystem für ein Netz mit Erdschlusskompensation bei einem einpoligen Erdschluss

$I_{\mathrm{F} \text { max }}$ maximaler Fehlerstrom für $\underline{Z}_{\mathrm{F}}=0$

Der Fehlerstrom $\underline{I}_{\mathrm{F}}$ ergibt sich zu:

$$
\underline{I}_{\mathrm{F}}=\frac{\underline{U}_{\mathrm{q}}}{\underline{Z}_{0} / 3+\underline{Z}_{\mathrm{F}}}
$$

Vernachlässigt man vorerst die Wirkung der Kabelschirme, so wirkt als Fehlerimpedanz $\underline{Z}_{F}$ einzig der Ausbreitungswiderstand der Fehlerstelle $R_{\mathrm{E}, \mathrm{F}}$. Der Fehlerstrom $\underline{I}_{\mathrm{F}}$ entspricht dem Erdungsstrom $I_{\mathrm{E}}$ und die Erdungsspannung $\underline{U}_{\mathrm{E}}$ der Fehlerstelle, ergibt sich mit Gl. 5:

$$
\underline{U}_{\mathrm{E}}=\underline{U}_{\mathrm{q}} \frac{R_{\mathrm{E}, \mathrm{F}}}{\underline{Z}_{0} / 3+R_{\mathrm{E}, \mathrm{F}}}
$$

Für ein 30-kV-Netz mit einem angenommenen maximalen Fehlerstrom von $67 \mathrm{~A}$ und einer maximalen Netzspannung $U_{\mathrm{n}}$ von $36 \mathrm{kV}$ ergibt sich eine Ersatzimpedanz im Nullsystem $\underline{Z}_{0}$ von $j 930 \Omega$. Beispielsweise ergibt dies gemeinsam mit einem Ausbreitungswiderstand $R_{\mathrm{E}, \mathrm{F}}$ von $1,4 \mathrm{k} \Omega$ einen Fehlerstrom $I_{\mathrm{F}}$ von $14,5 \mathrm{~A}$ und damit eine Erdungsspannung $U_{\mathrm{E}}$ von $20 \mathrm{kV}$, was ca. $98 \%$ der Quellspannung $\left(\left|\underline{U}_{\mathrm{q}}\right|=20,8 \mathrm{kV}\right)$ entspricht.

\subsection{Fehlerimpedanz unter Berücksichtigung geerdeter Kabelschirme}

Abb. 6 zeigt eine vereinfachte Netzstruktur im ländlichen Raum, welche im Weiteren für die Berechnung der Fehlerimpedanz $\underline{Z}_{\mathrm{F}}$ unter Berücksichtigung der Kabelschirme und des Erdungsstromes $I_{\mathbf{E}}$ verwendet wird.

Schirme von Mittelspannungskabeln werden üblicherweise an beiden Enden an Erdungsanlagen angeschlossen. Tritt ein Isolationsfehler im Kabel auf,

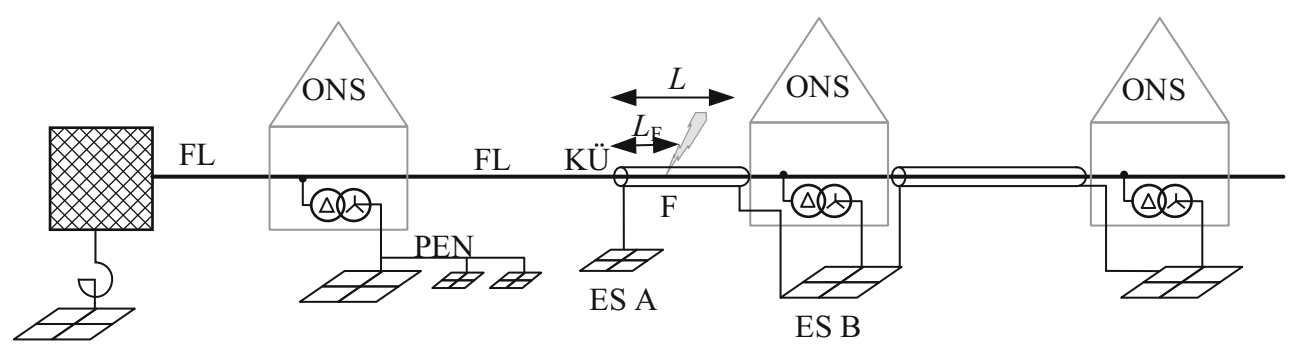

Abb. 6 Untersuchte ländliche Netzstruktur (ES Erdungssysteme, FL Freileitungen, KÜ Kabelaufführungsmast, ONS Ortsnetzstationen, $F$ Fehlerstelle, $L$ Länge des Kabels, $L_{F}$ Entfernung des Fehlers vom KÜ, PEN PEN-Leiter der Niederspannungsversorgung) 
führen die Kabelschirme einen Teil des Fehlerstromes zu den Erdungsanlagen, an welche sie angeschlossen sind (in Abb. 6, ES A und ES B). Dadurch wird die Fehlerimpedanz $Z_{\mathrm{F}}$ kleiner und der Fehlerstrom $I_{\mathrm{F}}$ steigt, jedoch verringert sich der Anteil des Stromes - der Erdungsstrom $I_{\mathrm{E}}$ - der an der Fehlerstelle F über den Ausbreitungswiderstand der Fehlerstelle $R_{\mathrm{E}, \mathrm{F}}$ in die Erde fließt und dort eine Potentialanhebung verursacht. Zusätzlich trägt die induktive Kopplung von Innenleiter und Schirm zu einer weiteren Reduktion des Erdungsstromes bei. Ist jedoch, wie hier untersucht, der Fehler in der Nähe einer vergleichsweise hochohmigen Erdungsanlage des Kabelaufführungsmastes, kommt dieser durch den Kabelreduktionsfaktor beschriebenen Effekt nur begrenzt zu tragen und wird in Folge vernachlässigt. Die Länge des Kabels zwischen den Ortsnetzstationen (ONS) und dem Kabelaufführungsmast beträgt häufig nicht mehr als $L=3 \mathrm{~km}$.

Die Erdungsimpedanz eines Kabelaufführungsmastes (KÜ, ES A) liegt häufig im Bereich von 2-10 $\Omega$, in Ausnahmefällen kann sie jedoch bis $\mathrm{zu} 100 \Omega$ betragen. Das zweite Ende des Kabelschirms ist an das Erdungssystem ES B der Ortsnetzstation (ONS) angeschlossen. Die Erdungsimpedanz $Z_{\mathrm{E}, \mathrm{B}}$ des Erdungssystems ES B der ONS ist meist wesentlich kleiner als die Erdungsimpedanz $Z_{\mathrm{E}, \mathrm{A}}$ des Erdungssystem ES A bei einem Kabelaufführungsmast, da an die Erdungsanlage einer ONS im Allgemeinen die PEN-Leiter und damit die Erder im Niederspannungsnetz angeschlossen sind.

In Österreich sind an die PEN-Leiter gemäß der Nullungsverordnung aus dem Jahre 1998 auch die Erder neuer Verbraucheranlagen anzuschließen, wenn der Verteilernetzbetreiber den betreffenden Netzbereich für die Anwendung der Nullung als Maßnahme des Fehlerschutzes in den angeschlossenen Niederspannungsanlagen freigegeben hat; auch die Umstellung bestehender Verbraucheranlagen auf die Nullung wurde vergleichsweise einfach ermöglicht.

Eine Kabelverbindung zur nächsten ONS wirkt bei geerdeten Kabelschirmen ebenfalls reduzierend auf die Erdungsimpedanz $Z_{\mathrm{E}, \mathrm{B}}$ von ES B. Im Allgemeinen liegt die Erdungsimpedanz einer ONS im Bereich von $0,5-5 \Omega$.

In Abb. 7 ist die Ersatzschaltung zur Bestimmung der Fehlerimpedanz $\underline{Z}_{\mathrm{F}}$ und des Erdungsstroms $\underline{I}_{\mathrm{E}}$ an der Fehlerstelle F dargestellt.

Ausgangspunkt für die Bestimmung des Erdungsstroms und der Schritt- und Berührungsspannungen für die verschiedenen Fälle in Abschn. 3.5 ist ein Basisszenario mit großteils typischen Werten. Ausgehend von diesem werden einzelne Parameter variiert. Die Parameter des Basisszenarios sind in Tab. 1 angegeben.

Ausgehend vom Basisszenario ist in Abb. 8 die Abhängigkeit des Erdungsstrom $I_{\mathrm{E}}$ vom spezifischen Bodenwiderstand $\varrho_{\mathrm{E}}$ und vom Übergangswiderstande $R_{\mathrm{B}}$ dargestellt. $R_{\mathrm{B}}=\infty \Omega$ entspricht dem Fall, dass keine

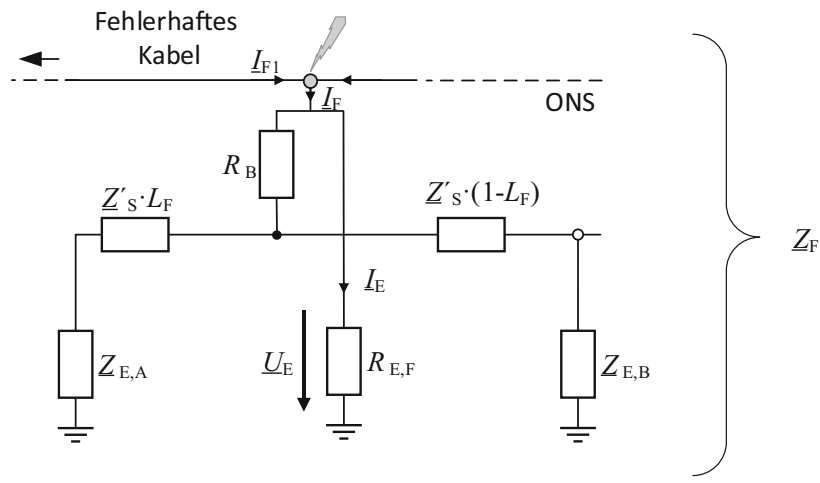

Abb. 7 Ersatzschaltung zur Bestimmung der Fehlerimpedanz $\underline{Z}_{\mathrm{F}}\left(R_{\mathrm{E}, \mathrm{F}}\right.$ Ausbreitungswiderstand der Fehlerstelle (abhängig vom örtlichen spezifischen Bodenwiderstand sowie der Fläche an der Übergangsstelle zum Erdreich), $R_{\mathrm{B}}$ Übergangswiderstand zwischen Kabelinnenleiter und -schirm (hauptsächlich bestimmt durch den Fehlerlichtbogen), $L_{F}$ Entfernung des Kabelfehlers vom Kabelaufführungsmast, $\underline{Z}_{\mathrm{E}, \mathrm{A}}$ Erdungsimpedanz der Erdungsanlage A, z. B. beim Kabelaufführungsmast, $\underline{Z}_{\mathrm{E}, \mathrm{B}}$ Erdungsimpedanz des Erdungssystems B, z. B. bei einer Ortnetzstation (ONS) einschließlich der Berücksichtigung weiterer über Kabelschirme und über PEN-Leiter verbundener Erdungssysteme, $\underline{Z}_{\mathrm{S}}^{\prime}$ Impedanz des Kabelschirms (etwa j0,6 $\Omega / \mathrm{km})$ )

\section{Tab. 1 Parameter des Basisszenarios}

\begin{tabular}{|l|l|}
\hline Fall No & 1 \\
\hline$U_{\mathrm{Netz}}$ & $30 \mathrm{kV}$ \\
\hline$U_{\mathrm{n}}$ & $36 \mathrm{kV}$ \\
\hline$I_{\mathrm{F}, \max }$ & $67 \mathrm{~A}$ \\
\hline$\rho_{\mathrm{E}}$ & $100 \Omega \mathrm{m}$ \\
\hline$A$ & $10 \mathrm{~cm}^{2}$ \\
$R_{\mathrm{B}}$ & $10 \Omega$ \\
\hline$Z_{\mathrm{E}, \mathrm{B}}$ & $2 \Omega$ \\
\hline$Z_{\mathrm{E}, \mathrm{A}}$ & $10 \Omega$ \\
\hline$L$ & $3 \mathrm{~km}$ \\
\hline$L_{\mathrm{F}}$ & $0,3 \mathrm{~km}$ \\
\hline
\end{tabular}

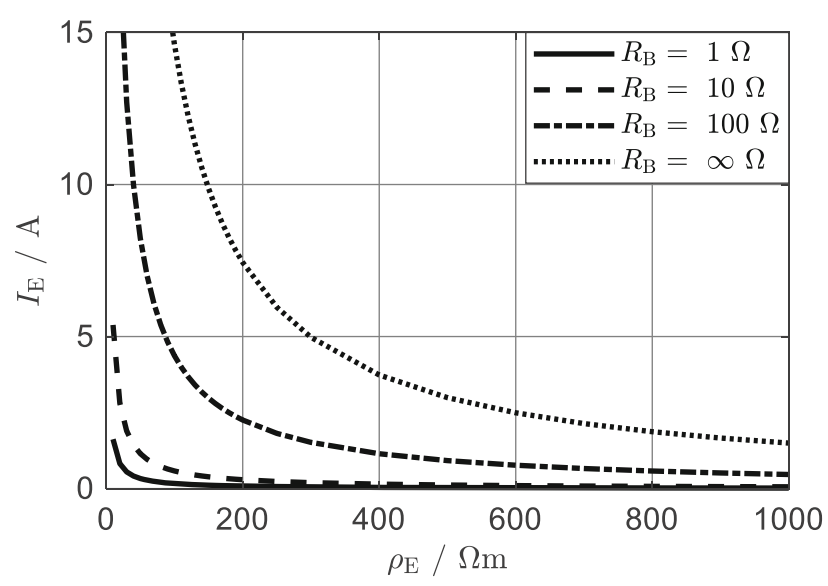

Abb. 8 Erdungsstrom $I_{E}$ in Abhängigkeit vom Übergangwiderstand $R_{\mathrm{B}}$ und vom spezifischen Bodenwiderstand $\varrho_{\mathrm{E}}$ für das Basisszenario 
Verbindung der Fehlerstelle zum Schirm angenommen wird.

Selbst für den ungünstigen Fall mit $R_{\mathrm{B}}=\infty \Omega$ und einen niedrigen spezifischen Bodenwiderstand $\varrho_{\mathrm{E}}$ von z.B. $50 \Omega \mathrm{m}$ liegt der Erdungsstrom $I_{\mathrm{E}}$ deutlich unter dem maximalen Fehlerstrom $I_{\mathrm{F} \text { max }}$ von $67 \mathrm{~A}$. Für das Basisszenario ergibt sich ein Erdungsstrom $I_{\mathrm{E}}=0,6 \mathrm{~A}$ und eine Erdungsspannung $U_{\mathrm{E}}=783 \mathrm{~V}$.

\subsection{Potentialverlauf an der Oberfläche}

Mit dem aus Abschn. 3.3 ermittelten Erdungsstrom $I_{\mathrm{E}}$ können der Verlauf des Potentials $\varphi$ an der Erdoberfläche sowie daraus die resultierenden Leerlauf-Spannungen $\left(U_{\mathrm{vT},} U_{\mathrm{vS}}, U_{\mathrm{vL}}\right)$ berechnet werden. Leerlauf-Berührungsspannungen $U_{\mathrm{vT}}$ können im Fall eines inneren Fehlers nur als Leerlauf-Liegestützspannungen $U_{\mathrm{vL}}$ auftreten, da die Fehlerstelle im Erdreich liegt und keine metallisch leitfähigen Teile zur Oberfläche führen.

Die Ausdehnung der Fehlerstelle ist im Vergleich zur Verlegetiefe $T$ des Kabels sehr klein $\left(\sqrt{\frac{A}{\pi}} \ll T\right)$. Die Potentialverteilung an der Erdoberfläche ist daher in guter Näherung von der Form des Erders unabhängig. Die Fehlerstelle kann somit hinreichend genau auch als Kugelerder in einer Tiefe $T$ angenommen werden. Damit können die bekannten Formeln für den Kugelerder zur Untersuchung verwendet werden [5]. Das an der Erdoberfläche auftretende Potenzial $\varphi$ der Fehlerstelle gegenüber Bezugserde kann folgendermaßen berechnet werden [5]:

$$
\varphi=\frac{I_{\mathrm{E}} \rho_{\mathrm{E}}}{2 \pi \sqrt{a^{2}+T^{2}}}
$$

mit:

$I_{\mathrm{E}} \quad$ Erdungsstrom in A

$\varrho_{\mathrm{E}} \quad$ spezifischer Widerstand des umgebenden Erdreiches in $\Omega \mathrm{m}$

$a$ horizontaler Abstand zur Fehlerstelle in m

$T$ vertikaler Abstand zwischen Oberfläche und Fehlerstelle in $\mathrm{m}$

Die maßgeblichen Schritt- und Liegestützspannungen im Leerlauf $\left(U_{\mathrm{vS}}, U_{\mathrm{vL}}\right)$ im horizontalen Abstand $a$ (siehe Abb. 2) werden aus Gl. 7 mit Gl. 8 bestimmt. Die Schrittweite $S$ beträgt für $U_{\mathrm{vS}}$ nach [2] $1 \mathrm{~m}$, für $U_{\mathrm{vL}}$ ergibt sich in Anlehnung an [4] $S=1,5 \mathrm{~m}$.

$$
\begin{aligned}
U_{\mathrm{v}}(a) & =\varphi\left(a-\frac{S}{2}\right)-\varphi\left(a+\frac{S}{2}\right) \\
& =\frac{I_{\mathrm{E}} \rho_{\mathrm{E}}}{2 \pi}\left(\frac{1}{\sqrt{(a-S / 2)^{2}+T^{2}}}-\frac{1}{\sqrt{(a+S / 2)^{2}+T^{2}}}\right)
\end{aligned}
$$

Der Potentialverlauf $\varphi$ und die Schritt- und Liegestützspannungen im Leerlauf $\left(U_{\mathrm{vS}}, U_{\mathrm{vL}}\right)$ an der Oberfläche hängen somit nach Gln. 7 und 8 vom Produkt $I_{\mathrm{E}} \varrho_{\mathrm{E}} \mathrm{ab}$.

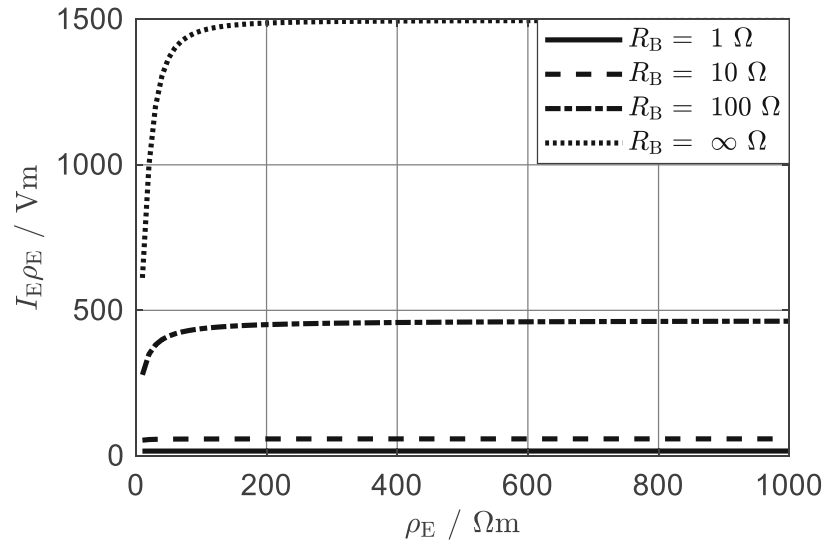

Abb. $9 l_{\mathrm{E} O \mathrm{E}}$ in Abhängigkeit vom Übergangswiderstand $R_{\mathrm{B}}$ und vom spezifischen Bodenwiderstand $\varrho_{E}$ für das Basisszenario

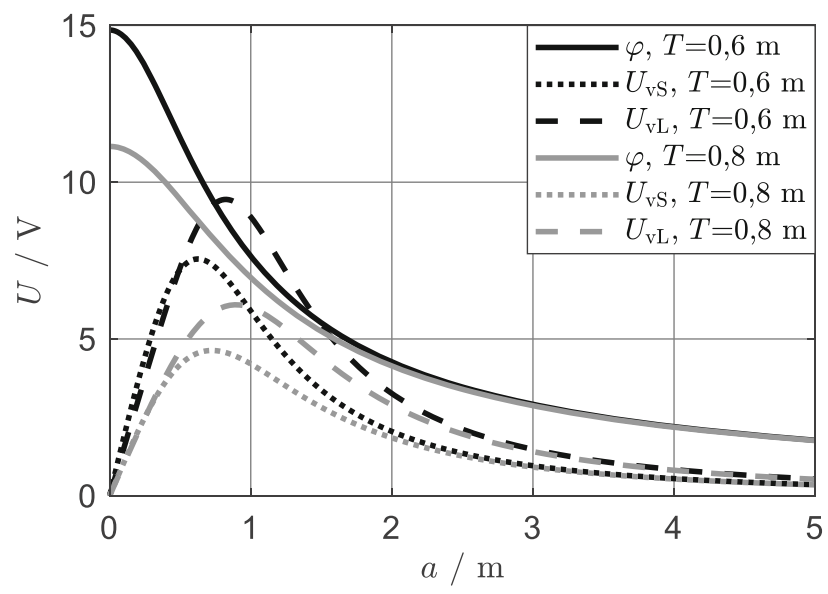

Abb. 10 Verlauf des Oberflächenpotentials $\varphi$ eines in der Tiefe $T=0,6 \mathrm{~m}$ verlegten Kabels sowie die Schrittspannungen $U_{\mathrm{vs}}$ und Leerlauf-Liegestützspannungen $U_{\mathrm{VL}}$ für verschiedene Verlegetiefen $T$ in Abhängigkeit des seitlichen Abstands zur Kabeltrasse a für das Basiszenario mit $I_{\mathrm{E}} \rho_{\mathrm{E}}=56 \mathrm{Vm}$

Für das mit den Werten aus Tab. 1 beschriebene Basisszenario ist in Abb. 9 unter Berücksichtigung der Kabelschirme für die Variation des Übergangwiderstandes $R_{\mathrm{B}}$ und des spezifischen Bodenwiderstands $\varrho_{\mathrm{E}}$ die Auswertung für den Ausdruck $I_{\mathrm{E}} \varrho_{\mathrm{E}}$ dargestellt.

Es ist ersichtlich, dass das Produkt $I_{\mathrm{E}} \varrho_{\mathrm{E}}$ im Wesentlichen für $\rho_{\mathrm{E}}>50 \Omega \mathrm{m}$ unabhängig von $\varrho_{E}$ ist. Für das Basisszenario mit $R_{\mathrm{B}}=10 \Omega$ ergibt sich $I_{\mathrm{E}} \rho_{\mathrm{E}}=56 \mathrm{Vm}$. In Abb. 10 sind der Verlauf des Oberflächenpotentials $\varphi$ gemäß Gl. 7 sowie die Schritt- und Liegestützspannungen im Leerlauf $\left(U_{\mathrm{vS}}, U_{\mathrm{vL}}\right)$ nach Gl. 8 für typische Verlegetiefen $T$ dargestellt. Die höchste Potentialanhebung über der Fehlerstelle beträgt hier 14,8 V. Die höchste Leerlauf-Liegestützspannung $U_{\mathrm{vL}}$ tritt bei einem horizontalen Abstand der Körpermitte (gemäß Abb. 2) von ca. 0,8 m seitlich der Kabeltrasse auf und liegt deutlich unter der zulässigen Berührungsspannung $U_{\text {Tp }}$ von $80 \mathrm{~V}$.

Abb. 11 zeigt in Abhängigkeit vom spezifischen Bodenwiderstand $\varrho_{E}$ die zulässige Leerlauf-Liege- 


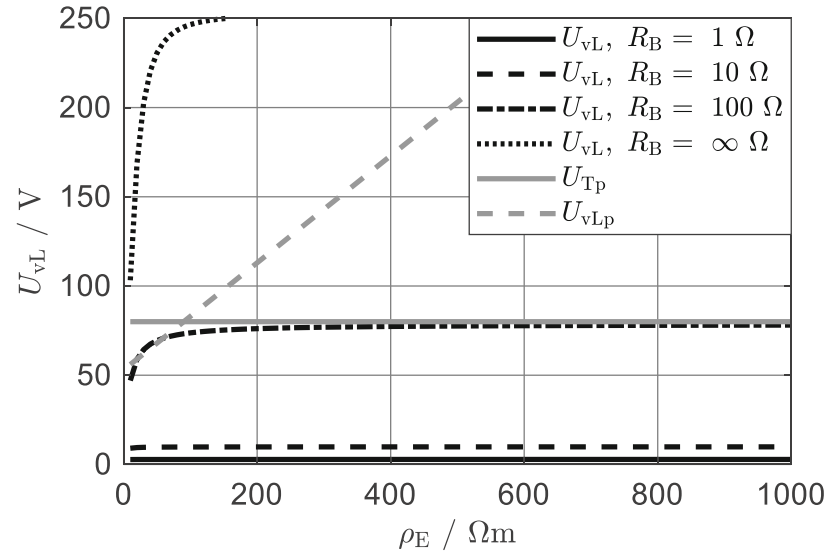

Abb. 11 Höchste auftretende Leerlauf-Liegestützspannungen $U_{\mathrm{VL}}$ für das Basiszenario und Verlegetiefe $T=0,6 \mathrm{~m}$ in Abhängigkeit vom spezifischen Bodenwiderstand $\varrho_{\mathrm{E}}$ und vom Übergangswiderstand $R_{\mathrm{B}}, U_{\mathrm{vLp}}$ zulässige Leerlauf-Liegestützspannung

stützspannung $U_{\text {vLp }}$ sowie die für verschiedene Übergangswiderstände $R_{\mathrm{B}}$ auftretenden höchsten Leerlauf-Liegestützspannungen $U_{\mathrm{vL}}$. Man erkennt, dass nur in Ausnahmefällen $\left(R_{\mathrm{B}}=\infty\right)$ die Leerlauf-Berührungsspannung für liegende Personen $U_{\text {vLp }}$ überschritten wird und die zulässige Berührungsspannung $U_{\mathrm{Tp}}=80 \mathrm{~V}$ überschritten ist.

\subsection{Szenarien}

Für eine umfassende Untersuchung werden neben dem zuvor beschriebenen Basiszenario (Fall 1) auch mehrere andere Szenarien untersucht. Die Parameter und Ergebnisse sind zusammenfassend in Tab. 2 dargestellt. Nach [2] ist das Risiko einer Personenge- fährdung vertretbar, wenn die auftretenden Schrittund Berührungsspannungen (hier $U_{\mathrm{vs}}, U_{\mathrm{vL}}$ ) die zulässige Berührungsspannung $U_{\mathrm{Tp}}=80 \mathrm{~V}$ bzw. die entsprechende zulässige Leerlauf-Berührungsspannung nicht überschreiten.

- Fall 1 (Basisszenario): Für das beschriebene Basiszenario ergibt sich ein Fehlerstrom $I_{\mathrm{F}}$ beinahe in der Höhe des maximalen Fehlerstroms $I_{\mathrm{F} \text { max }}$ von $67 \mathrm{~A}$, jedoch beträgt der für die Potentialanhebung relevante Erdungsstrom $I_{\mathrm{E}}$ nur etwa 1/100 des Fehlerstroms. Für die höchste Leerlauf-Schrittspannung $U_{v S}$ ergibt sich 7,5V, für die Leerlauf-Liegestützspannung $U_{\mathrm{vL}} 9,4 \mathrm{~V}$. Sie liegen damit deutlich unter der zulässigen Berührungsspannung $U_{\mathrm{Tp}}$ von $80 \mathrm{~V}$ bzw. der zulässigen Leerlauf-Liegestützspannung von $U_{\mathrm{vLp}}=73 \mathrm{~V}$.

- Fall 2 und 3: In diesen Fällen wird der Fehlerort $L_{\mathrm{F}}$ variiert. Dies hat im Vergleich zum Basisszenario keine wesentlichen Auswirkungen auf die Schrittund Liegestützspannungen.

- Fall 4 und 5: Hier wird die Erdungsimpedanz der Erdungssysteme des Kabelaufführungsmastes $Z_{\mathrm{E}, \mathrm{A}}$ und der Ortsnetzstation $Z_{\mathrm{E}, \mathrm{B}}$ gegenüber dem Basisszenario (Fall 1) auf maximal zu erwartende Werte erhöht. Im Fall 5 sind beide Erdungsimpedanzen erhöht; selbst hier sind die höchsten Leerlauf-Liegestützspannungen $U_{\mathrm{vL}}$ von $11,8 \mathrm{~V}$ weit unter dem zulässigen Wert $U_{\mathrm{Tp}}=80 \mathrm{~V}$.

- Fall 6: Die Berechnung wurde hier mit einer NetzNennspannung von $20 \mathrm{kV}$ und daher mit einem höchsten Erdschlussreststrom von $60 \mathrm{~A}$. gerechnet. Die maximale Leerlauf-Liegestützspannung ist um $11 \%$ niedriger als beim Basisszenario mit $30 \mathrm{kV}$.

Tab. 2 Berechnungsergebnisse für die Fälle 1-10 mit Variation einzelner Parameter (fett gekennzeichnet), Verlegetiefe der Kabel $T=0,6 \mathrm{~m}$

\begin{tabular}{|c|c|c|c|c|c|c|c|c|c|c|}
\hline Fall No & 1 & 2 & 3 & 4 & 5 & 6 & 7 & 8 & 9 & 10 \\
\hline$U_{\text {Netz }}$ in $\mathrm{kV}$ & 30 & 30 & 30 & 30 & 30 & 20 & 30 & 30 & 30 & 30 \\
\hline$U_{\mathrm{n}}$ in $\mathrm{kV}$ & 36 & 36 & 36 & 36 & 36 & 24 & 36 & 36 & 36 & 36 \\
\hline$I_{F, \text { max }}$ in $A$ & 67 & 67 & 67 & 67 & 67 & 60 & 10 & 10 & 67 & 67 \\
\hline$L_{F} / L$ in $p u$ & 0,1 & 0,5 & 0,9 & 0,1 & 0,1 & 0,1 & 0,1 & 0,1 & 0,1 & 0,1 \\
\hline$\rho \mathrm{E} i n \Omega m$ & 100 & 100 & 100 & 100 & 100 & 100 & 100 & 100 & 100 & 100 \\
\hline$R_{\mathrm{B}}$ in $\Omega$ & 10 & 10 & 10 & 10 & 10 & 10 & 10 & 5 & 100 & $\infty$ \\
\hline $\mathrm{Z}_{\mathrm{E}, \mathrm{B}}$ in $\Omega$ & 2 & 2 & 2 & 2 & 5 & 2 & 2 & 2 & 2 & 2 \\
\hline$Z_{\mathrm{E}, \mathrm{A}}$ in $\Omega$ & 10 & 10 & 10 & 100 & 100 & 10 & 10 & 2 & 100 & 100 \\
\hline$R_{\mathrm{E}, \mathrm{F}}$ in $\mathrm{k} \Omega$ & 1,40 & 1,40 & 1,40 & 1,40 & 1,40 & 1,40 & 1,40 & 1,40 & 1,40 & 1,40 \\
\hline$Z_{F}$ in $\Omega$ & 11,7 & 11,6 & 11,6 & 12,0 & 14,8 & 11,7 & 11,7 & 6,1 & 95,1 & 1401 \\
\hline IF in $\mathrm{A}$ & 66,7 & 66,8 & 66,9 & 66,6 & 66,5 & 59,6 & 10,0 & 10,0 & 62,6 & 12,2 \\
\hline$A_{E}$ in $A$ & 0,6 & 0,6 & 0,6 & 0,6 & 0,1 & 0,5 & 0,1 & 0,0 & 4,2 & 12,2 \\
\hline$l_{E} \rho_{E}$ in $A \Omega m$ & 56 & 55 & 55 & 57 & 70 & 50 & 8 & 4 & 425 & 1216 \\
\hline$U_{E}$ in $V$ & 783 & 776 & 774 & 797 & 988 & 700 & 117 & 61 & $6 k$ & $20 \mathrm{k}$ \\
\hline $\operatorname{Max}(\varphi)$ in V & 14,8 & 14,7 & 14,7 & 15,1 & 18,7 & 13,2 & 2,2 & 1,2 & 112,7 & 322,4 \\
\hline $\operatorname{Max}\left(U_{v S}\right)$ in V & 7,5 & 7,5 & 7,4 & 7,7 & 9,5 & 6,7 & 1,1 & 0,6 & 57,3 & 164 \\
\hline $\operatorname{Max}\left(U_{v L}\right)$ in V & 9,4 & 9,3 & 9,3 & 9,6 & 11,8 & 8,4 & 1,4 & 0,7 & 71,7 & 205 \\
\hline$U_{V L p}$ in $V$ & 73 & 73 & 73 & 73 & 73 & 73 & 73 & 73 & 73 & 73 \\
\hline
\end{tabular}


- Fall 7 und 8: Die in diesen beiden Fälle beschriebenen Szenarien, welche typische Betriebsfälle darstellen. D.h. bei diesen Fällen sind für den Fehlerstrom und für die Erdungsimpedanzen typische Werte statt der vergleichsweise hohen Werte beim Basisszenario angenommen. Der Fehlerstrom ist mit $10 \mathrm{~A}$ angenommen. Fall 7 berücksichtigt dabei den Fall einer Verbindung der Kabelschirme zwischen einem Kabelaufführungsmast und einer Ortsnetzstation, während Fall 8 einer Verbindung der beiden Enden der Kabelschirme mit einer gut geerdeten Ortsnetzstation entspricht. Die LeerlaufLiegestützspannungen $U_{\mathrm{vL}}$ sinken dabei im Vergleich zu Fall 1 auf sehr geringe Werte von 1,4 V bzw. $0,7 \mathrm{~V}$

- Fall 9 und 10 sind Extremszenarien mit einer sehr schlechten (Fall 9) oder einer fehlenden (Fall 10) Verbindung des Kabelschirmes zu den benachbarten Erdungsanlagen, sowie weiters einem örtlich niedrigen spezifischen Bodenwiderstand. Bei Fall 9 wird die zulässige Leerlauf-Berührungsspannung $U_{\text {vLp }}$ unterschritten, während sie bei Fall 10 rechnerisch überschritten wird. Im Fall 10 ergibt sich die Erdungsspannung mit $17 \mathrm{kV}$, bei der ein Lichtbogen zum Kabelschirm jedenfalls zünden würde. Bei den zumindest einseitig geerdeten Kabelschirmen von Mittelspannungskabeln kann dieser Fall 10 daher gar nicht auftreten.

In den Fällen 1-9 wird die zulässige Berührungsspannung $U_{\mathrm{Tp}}=80 \mathrm{~V}$ bzw. die Leerlauf-Liegestützspannung $U_{\text {vLp }}$ nicht überschritten. Der Fall 10 ist nicht praxisrelevant (siehe oben). Gemäß [2] ist daher im Nahbereich des Kabelfehlers das Risiko einer Gefährdung von Personen vertretbar.

\section{Potentialverschleppung durch Begleiterder}

Ein weiteres hier zu betrachtendes Szenario im Bereich von Mittelspannungskabeln besteht bei Erdschlüssen in Ortsnetzstationen bzw. Kabelführungsmasten. Hier können allfällige Begleiterder der Kabel Oberflächenpotentiale und damit Liegestützspannungen und Schrittspannungen im Bereich der Kabeltrasse verursachen. In Abschn. 4.1 wird das für die Berechnungen verwendete Modell erläutert, in Abschn. 4.2 die relevante Erdungsimpedanz für verschiedene Fälle untersucht, um so die ungünstigsten Fälle und die Einflussparameter analysieren zu können. Für den ungünstigsten Fall hinsichtlich der Erdungsspannung werden in Abschn. 4.3 die Oberflächenpotentiale und daraus die Schritt- und Liegestützspannungen entlang der Kabeltrasse mit den Begleiterdern ermittelt.

„Klassische“ Berührungsspannungen $U_{\mathrm{T}}$ im Bereich des Begleiterders könnten nur auftreten, wenn der Begleiterder direkt berührbar wäre oder eine Verbindung mit anderen geerdeten, leitfähigen Objekten, die von der Erdoberfläche aus berührbar sind, bestehen würde. Dies wird hier nicht näher betrachtet.

\subsection{Modellbildung}

Bei Mittelspannungskabeln werden häufig Bänder oder Runddrähte aus verzinktem Stahl als Begleiterder mitverlegt. Diese Begleiterder reduzieren die Erdungsimpedanz im Bereich der Ortsnetzstationen und haben - da sie über dem Kabel verlegt werden - eine Schutzwirkung hinsichtlich atmosphärischer Einwirkungen (Blitzeinschlägen).

Zwei typische Verlegearten können unterschieden werden:

- Der Begleiterder wird durchgängig über die gesamte Länge des Kabels mitverlegt und an beiden Enden mit den Erdungssystemen, an die auch die Kabelschirme angeschlossen werden, verbunden (Abb. 12a).

- Von beiden Kabelenden wird je ein Bund Erdband - entspricht in der Regel etwa $50 \mathrm{~m}$ - mitverlegt (Abb. 12b).

Das Erdungssystem ES A stellt analog zum Abschn. 3.4 die Erdungsanlage eines Kabelaufführungsmastes dar, das Erdungssystem ES B repräsentiert die Erdungsanlage einer Ortsnetzstation.

Die Fehlerimpedanz $\underline{Z}_{\mathrm{F}}$ ist durch die Gesamtimpedanz der verbundenen Erdungssysteme gegeben. Sie ist durch den Begleiterder nun wesentlich niederohmiger als die Ersatzimpedanz des Netzes (siehe Abschn. 3.2). Damit ist eine Modellierung des speisenden Netzes als Stromquelle gerechtfertigt. Es wird daher bei der Untersuchung von einem eingeprägten Strom ausgegangen. In das Erdungssystem ES A wird entsprechend einem Fehlerfall am Kabelaufführungsmast der maximale Fehlerstrom von $I_{\mathrm{F}, \max }=67$ A eingespeist.

Für die Berechnung der Erdungsimpedanzen als auch der Schritt- und Berührungsspannungen wird das Programm XGSLab ${ }^{\mathrm{TM}}$, Modul XGSA FD [6] verwendet. Das Modul XGSA FD erlaubt es, die Längsimpedanzen der Kabelschirme und der Begleiterder sowie Kopplungen zwischen diesen Leitern zu berücksichtigen. Die Berücksichtigung der Längsimpedanzen ist
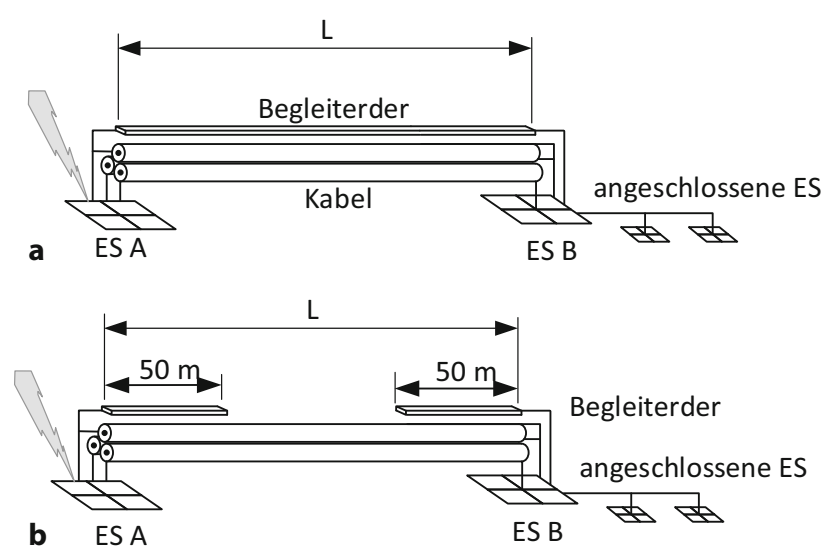

Abb. 12 Verlegung von Begleiterdern, (a): durchgängig, (b): beiderseits jeweils $50 \mathrm{~m}$ weit 
wegen der verhältnismäßig großen Ausdehnung des durchgehenden Begleiterders von Bedeutung.

ES A und ES B werden vereinfacht als Ringerder um ein Quadrat mit einer Seitenlänge von $2 \mathrm{~m}$ modelliert. Die an das ES B angeschlossenen Erdungssysteme werden mit Hilfe einer zusätzlichen Impedanz $\underline{Z}_{\text {E,B PEN }}$ berücksichtigt. Die typische Länge des Kabels wird mit $500 \mathrm{~m}$ angenommen.

\subsection{Gesamterdungsimpedanz}

Für die Untersuchung der Auswirkungen der Begleiterder im Zusammenhang mit Erdschlüssen in Ortsnetzstationen bzw. Kabelführungsmasten werden im Folgenden Szenarien mit unterschiedlichen Verbindungen zwischen den erdfühligen Elementen berücksichtigt. Dabei wird die gesamte Erdungsimpedanz der verbundenen Erdungsanlagen bestimmt, um die Abhängigkeit von der Verbindungsart zu zeigen. Der spezifische Bodenwiderstand wird in allen Fällen vorerst einheitlich mit $100 \Omega \mathrm{m}$ angenommen.

Folgende Fälle werden untersucht und in Tab. 3 zusammenfassend dargestellt:

- Fall A: Hier wird nur das Erdungssystem ES A der Ortsnetzstation alleine berücksichtigt, d.h. es sind hier weder Kabelschirme noch Begleiterder angeschlossen. Der Betrag der Erdungsimpedanz $\underline{Z}_{\mathrm{F}}$ ergibt sich zu 19,2 $\Omega$.

- Fälle B-C: An ES A werden Begleiterder angeschlossen, im Fall B mit einer Länge von $50 \mathrm{~m}$ und im Fall C mit $500 \mathrm{~m}$. Der Betrag der Gesamterdungsimpedanz $\underline{Z}_{\mathrm{F}}$ sinkt dadurch auf 3,6 $\Omega$ bzw. $1,15 \Omega$. Bemerkenswert ist der bereits stark induktive Anteil mit einem Winkel von $19^{\circ}$ bei einem $500 \mathrm{~m}$ langen Begleiterder.

- Fälle D-F: Diese beiden Fälle zeigen den Einfluss der Verbindung der beiden Erdsysteme ES A und ES B über den gegen Erde isolierten Kabelschirm, jedoch als Mittelspannungskabel ohne Begleiterder. Im Fall D führt dies zu einer Halbierung von $\underline{Z}_{\mathrm{F}}$ im Vergleich zu Fall A. Die Fälle E und F berücksichtigen über PEN-Leiter oder Kabelschirme angeschlossene Impedanzen mit $\underline{Z}_{\mathrm{E}, \mathrm{B} \text { _PEN }}$. Mit einer Impedanz $\underline{Z}_{\mathrm{E}, \mathrm{B} \text { PEN }}$ von $10 \Omega \angle 0^{\circ}$ (Fall E) entsprechend dem typischen Ausbreitungswiderstandes eines einzelnen Einfamilienhauses sinkt der Betrag der Gesamterdungsimpedanz $\underline{Z}_{\mathrm{F}}$ bereits auf rund $5 \Omega$. Bei Fall F mit $\underline{Z}_{\mathrm{E}, \mathrm{B} \_\mathrm{PEN}}=2 \Omega \angle 0^{\circ}$ (entspricht in etwa der Erdungswirkung der Fundamenterder von 5 Wohnhäusern) ergibt sich ein $\underline{Z}_{\mathrm{F}}=1,79 \Omega \angle 8^{\circ}$.
- Fälle G-I: Bei Fall G und Fall H sind zusätzlich 50 m lange Begleiterder an ES A und ES B angeschlossen. Dadurch sinkt die Erdungsimpedanz weiter ab. Im Vergleich zu Fall B ohne Verbindung der Kabelschirme halbiert sich die Impedanz im Fall G bzw. sinkt im Fall $\mathrm{H}$ der Betrag der Impedanz auf $0,6 \Omega$ wenn $\underline{Z}_{\mathrm{E}, \mathrm{B} P \mathrm{PEN}}=2 \Omega \angle 0^{\circ}$ beträgt. Fall J hat im Vergleich $\mathrm{zu}$ Fall $\mathrm{H}$ ein ohmsch-induktives $\underline{Z}_{\mathrm{E}, \mathrm{B} \text { PEN }}=2 \Omega \angle 45^{\circ}$, dabei steigt die Gesamtimpedanz auf $1,19 \Omega \angle 45^{\circ}$.

- Fälle J-K: Diese Fälle zeigen Ähnliches wie die Fälle G-I, nur ist angenommen, dass durchgehend Begleiterder verlegt sind. Im Fall K bringt der durchgehende Begleiter gegenüber den beiden $50 \mathrm{~m}$ langen Begleiterderabschnitten (Fall $\mathrm{H}$ ) in Bezug auf die Erdungsimpedanz kaum einen Vorteil, da die Erdungsimpedanz wesentlich von der Verbindung zu den PEN-Leitern des Niederspannungsverteilernetzes bestimmt wird.

Für die weitere Überlegungen wird vom Fall G ausgegangen, bei dem am Mittelspannungskabel an beiden Seiten ein $50 \mathrm{~m}$ langer Begleiterder mitverlegt ist. Diese Auswahl ist dadurch begründet, dass einerseits die Auswirkung der Begleiterder auf die Schritt- und Liegestützspannungen untersucht werden soll und andererseits dieser Fall hinsichtlich der Erdungsimpedanz den ungünstigsten der betrachteten Fälle der Mittelspannungskabel mit Begleiterdern darstellt.

Die Erdungsimpedanz bei Fall $G$ steigt bei einem spezifischen Bodenwiderstands $\rho_{\mathrm{E}}=1000 \Omega \mathrm{m}$ auf $\underline{Z}_{\mathrm{F}}=$ $17,8 \Omega$. Dies bedeutet, das selbst für hohe spezifische Bodenwiderstände die Erdungsimpedanz im Vergleich zur Ersatzimpedanz des Netzes (siehe Abschn. 3.2) gering ist, und dadurch die Vorgangsweise mit dem eingeprägten Strom $I_{\mathrm{F} \text { max }}$ auch für höhere spezifische Bodenwiderstände anwendbare Werte ergibt. Daher wird die weitere Untersuchung in diesem Abschnitt mit eingeprägtem Strom durchgeführt.

\subsection{Oberflächenpotentiale, Schritt- und Liegestützspannungen}

Für den Fall G sind in Abb. 13 die Isolinien des Potentials $\varphi$ an der Erdoberfläche, in Abb. 14 die Isolinien der Leerlauf-Schrittspannungen $U_{\mathrm{vS}}$ und in Abb. 15 die Isolinien der Leerlauf-Liegestützspannungen $\mathrm{U}_{\mathrm{vL}}$ im Bereich des Begleiterders angrenzend an das Erdungssystem A dargestellt. Der Begleiterder liegt bei $y=0 \mathrm{~m}$ in einer Tiefe $0,6 \mathrm{~m}$ und verläuft von $x=0 \mathrm{~m}$ bis $x=50 \mathrm{~m}$. Die Erdungsanlage ES A liegt im Bereich

Tab. 3 Erdungsimpedanzen $Z_{\mathrm{F}}$ für die Szenarien mit den Begleiterdern

\begin{tabular}{|l|l|l|l|l|l|l|l|l|l|l|l|l|l|l}
\hline Fall & A & B & C & D & E & F & G & H & I & J & $K$ \\
\hline Begleiterder & Nein & $50 \mathrm{~m}$ & $500 \mathrm{~m}$ & Nein & Nein & Nein & $50 \mathrm{~m}$ & $50 \mathrm{~m}$ & $50 \mathrm{~m}$ & $500 \mathrm{~m}$ & $500 \mathrm{~m}$ \\
\hline Schirm angeschlossen & Nein & Nein & Nein & Ja & Ja & Ja & Ja & Ja & Ja & Ja & Ja \\
\hline$\underline{Z}_{\mathrm{E}, \mathrm{B} \_ \text {PEN }}$ in $\Omega$ & $\infty$ & $\infty$ & $\infty$ & $\infty$ & 10 & 2 & $\infty$ & 2 & 2 & $\infty$ & 2 \\
\hline & & & & & $\angle 0^{\circ}$ & $\angle 0^{\circ}$ & & $\angle 0^{\circ}$ & $\angle 45^{\circ}$ & & $\angle 0^{\circ}$ \\
\hline$\underline{Z}_{\mathrm{F}}$ in $\Omega$ & 19,2 & 3,60 & 1,15 & 9,46 & 4,98 & 1,79 & 1,86 & 0,59 & 1,19 & 0,74 & 0,59 \\
& $\angle 0^{\circ}$ & $\angle 1^{\circ}$ & $\angle 19^{\circ}$ & $\angle 0^{\circ}$ & $\angle 2^{\circ}$ & $\angle 8^{\circ}$ & $\angle 3^{\circ}$ & $\angle 9^{\circ}$ & $\angle 25^{\circ}$ & $\angle 16^{\circ}$ & $\angle 18^{\circ}$ \\
\hline
\end{tabular}


Abb. 13 Isolinien des Oberflächenpotentials $\varphi$ in V im Bereich des Begleiterders von $x=0-50 \mathrm{~m}$

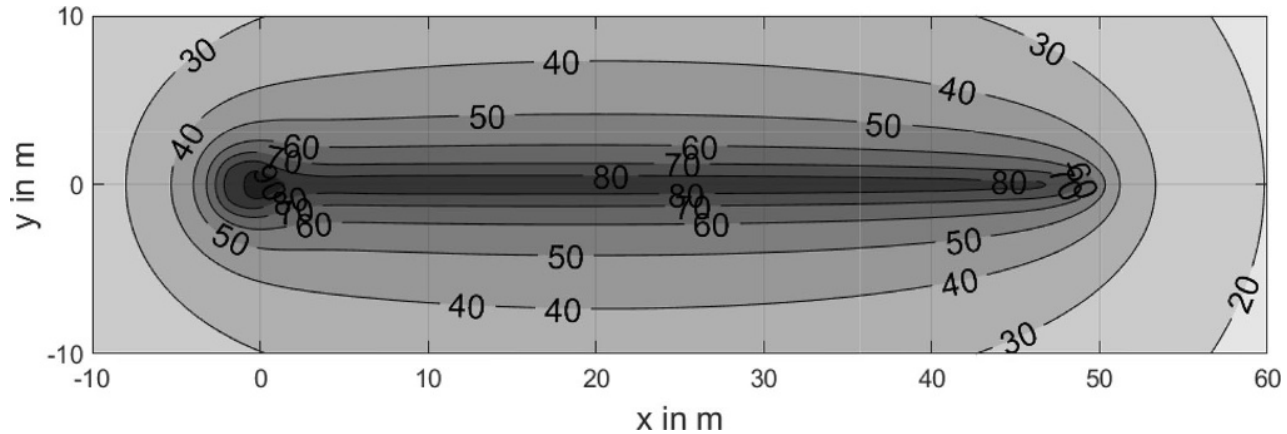

Abb. 14 Isolinien der Leerlauf-Schrittspannungen $U_{v}$ sin $V$ im Bereich über dem Begleiterder (von $x=0-50 \mathrm{~m})$

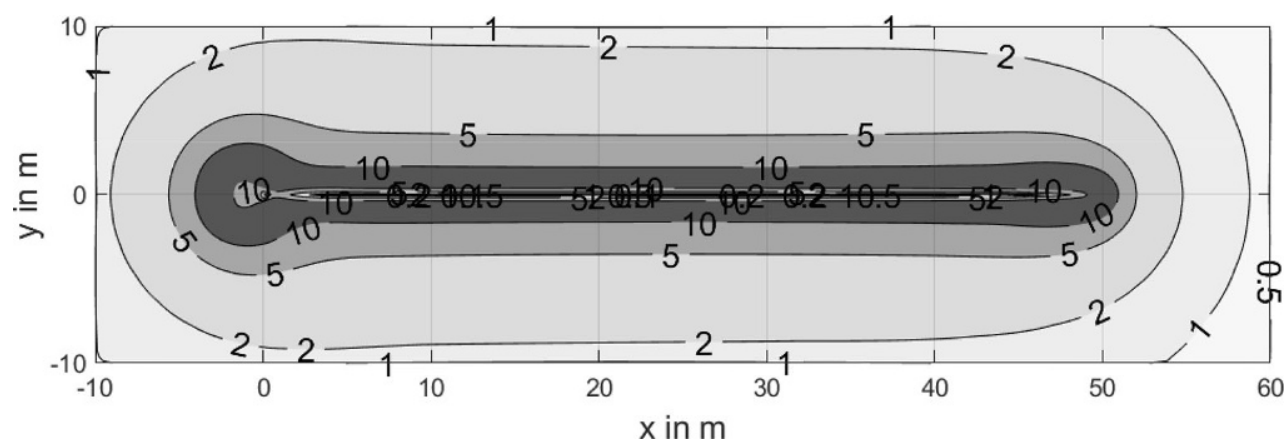

Abb. 15 Isolinien der Leerlauf-Liegestützspannungen $U_{\mathrm{vL}}$ in $\mathrm{V}$ im Bereich über dem Begleiterder (von $x=0-50 \mathrm{~m}$ )

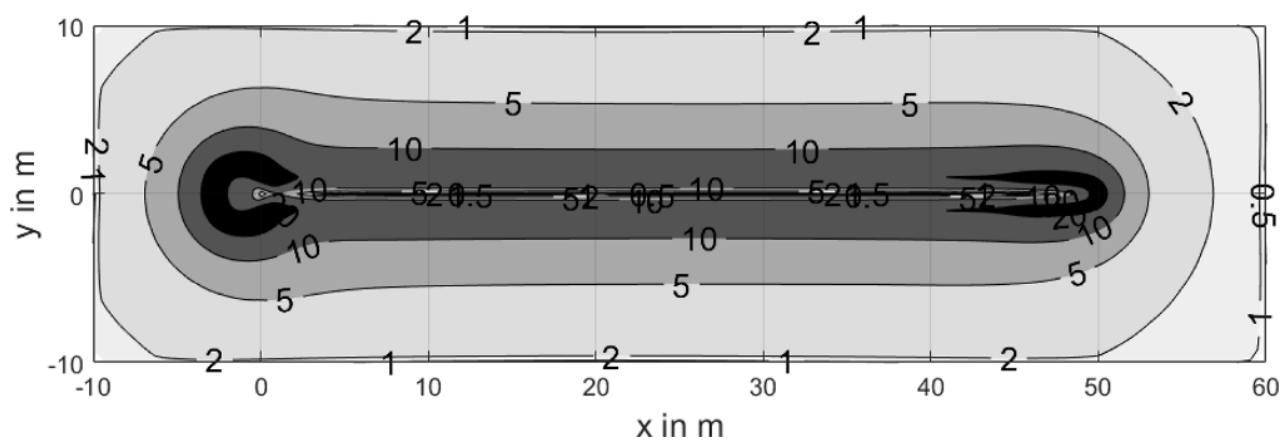

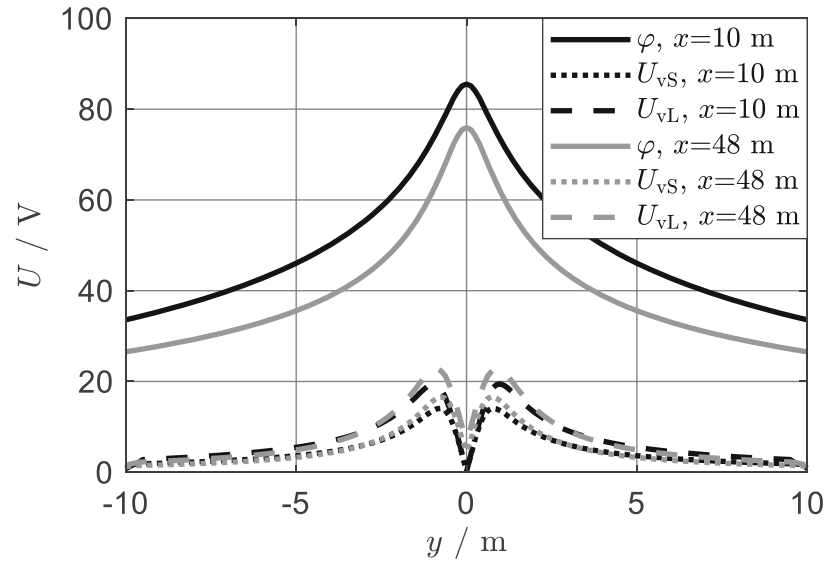

Abb. 16 Oberflächenpotential $\varphi$, Leerlauf-Schrittspannungen $U_{\mathrm{vs}}$ und Leerlauf-Liegestützspannungen $U_{\mathrm{vL}}$ quer über dem Begleiterder für verschiedenen Positionen $x$ entlang der Kabeltrasse $x=-2 \mathrm{~m}$ bis $x=0 \mathrm{~m}$. Wie in Abb. 14 und Abb. 15 ersichtlich, treten die höchsten Schritt- und Liegestützspannungen am Ende des Begleiterders auf. In Abb. 16 sind das Oberflächenpotential $\varphi$, die Leerlauf-Schrittspannungen $U_{\mathrm{vS}}$ und die Leerlauf-Liegestützspannungen $U_{\mathrm{vL}}$ quer über dem Begleiterder für verschiedenen Positionen $x$ dargestellt.

Die höchsten auftretenden Leerlauf-Schritt- und Leerlauf-Liegestützspannungen bei $\rho_{\mathrm{E}}=100 \Omega \mathrm{m}$ betragen $U_{\mathrm{vS}}=16,7 \mathrm{~V}$ und $U_{\mathrm{vL}}=22,9 \mathrm{~V}$ und liegen weit unter der zulässigen Berührungsspannung $U_{\mathrm{Tp}}=80 \mathrm{~V}$.

Für einen vergleichsweise hohen spezifischen Bodenwiderstand von $\rho_{\mathrm{E}}=1000 \Omega \mathrm{m}$ ergibt sich eine höchste Leerlauf-Schrittspannung $U_{\mathrm{vS}}=164 \mathrm{~V}$ und eine höchste Leerlauf-Liegestützspannung $U_{\mathrm{vL}}=228 \mathrm{~V}$. Die zulässige Leerlauf-Liegestützspannung von $208 \mathrm{~V}$ gemäß Abschn. 2 wird dabei geringfügig überschritten. Für solch eine Konfiguration mit sehr hohen spezifischen Bodenwiderständen $\rho_{\mathrm{E}} \geq 1000 \Omega \mathrm{m}$ sind für die tatsächlich vorherrschenden Gegebenheiten genauere Untersuchungen und gegebenenfalls Maßnahmen notwendig, die dazu führen, dass die zulässigen 
Leerlauf-Liegestützspannungen nicht überschritten werden.

Somit ist auch für den Fall eines Fehlers bei einem Kabelübergangsmast bzw. einer Ortsnetzstation gezeigt, dass in den meisten Fällen $\left(\rho_{\mathrm{E}}<1000 \Omega \mathrm{m}\right)$ die Spannungsverschleppungen über Kabelbegleiterder $\mathrm{zu}$ keinen unzulässigen Schritt- und Berührungsspannungen im Bereich der Mittelspannungskabel führen.

\section{Zusammenfassung}

In erdschlusskompensierten Mittelspannungsnetzen mit Nennspannungen bis $30 \mathrm{kV}$ werden Schritt- und Berührungsspannungen bei Kabelfehlern und Fehlern bei Kabelaufführungsmasten bei Annäherung der Kabeltrassen an Swimmingpools untersucht.

Bei Kabelfehlern an Mittelspannungskabeln treten keine unzulässigen Leerlauf-Schrittspannungen $U_{v S}$ und Leerlauf-Liegestützspannungen für $U_{\mathrm{vL}}$ auf, da der Anteil des für die Potentialanhebung verantwortlichen Stromes mit steigendem Ausbreitungswiderstand der Fehlerstelle $R_{\mathrm{E}, \mathrm{F}}$ bzw. durch die an Erdungsanlagen angeschlossenen Kabelschirme deutlich absinkt. Selbst bei Extremszenarien sind in praktischen Fällen keine gefährlichen Spannungen zu erwarten. Dabei wird berücksichtigt, dass sich Personen in der Nähe von Swimmingpools auch barfuß aufhalten.

Begleiterder führen im Falle eines Erdschlusses bei einer Ortsnetzstation oder auch im kritischeren Fall eines Fehlers bei einem Kabelaufführungsmasten zu Potentialverschleppungen. Es sind in den meisten Fällen hier keine gefährlichen Schritt- oder Berührungsspannungen zu erwarten. Für Gebiete mit sehr hohen spezifischen Bodenwiderständen $\left(\rho_{\mathrm{E}} \geq 1000 \Omega \mathrm{m}\right)$ ist eine detailliertere Untersuchung der tatsächlich auftretenden Liegenstütz-Spannungen notwendig und es sind gegebenenfalls Maßnahmen zu ergreifen, um das Risiko einer Gefährdung auf ein vertretbares Maß $\mathrm{zu}$ reduzieren. Weiters ist festzuhalten, dass ein Szenario mit dem direkten Berühren eines Begleiterders im Bereich eines Swimmingpools als nicht realistisch einzuschätzen ist, und daher nicht weiter untersucht wurde.

Es sind daher im Zusammenhang mit der Annäherung von Mittelspannungskabeln an Swimmingpools im Allgemeinen bei den untersuchten Szenarien keine besonderen Maßnahmen zur Verringerung von Liegestütz- und Schrittspannungen erforderlich.

Danksagung Für die fachlichen Diskussionen danken die Autoren Dr. Christoph Groiß, Ing. Wolfgang Reisinger, DI Gerhard Krobath, DI Walter Hipp, DI Johannes Wisiak und Dr. Franz Strempfl.

Funding Open access funding provided by Graz University of Technology.

Open Access Dieser Artikel wird unter der Creative Commons Namensnennung 4.0 International Lizenz veröffentlicht, welche die Nutzung, Vervielfältigung, Bearbeitung, Verbreitung und Wiedergabe in jeglichem Medium und Format erlaubt, sofern Sie den/die ursprünglichen Autor(en) und die Quelle ordnungsgemäß nennen, einen Link zur Creative Commons Lizenz beifügen und angeben, ob Änderungen vorgenommen wurden.

Die in diesem Artikel enthaltenen Bilder und sonstiges Drittmaterial unterliegen ebenfalls der genannten Creative Commons Lizenz, sofern sich aus der Abbildungslegende nichts anderes ergibt. Sofern das betreffende Material nicht unter der genannten Creative Commons Lizenz steht und die betreffende Handlung nicht nach gesetzlichen Vorschriften erlaubt ist, ist für die oben aufgeführten Weiterverwendungen des Materials die Einwilligung des jeweiligen Rechteinhabers einzuholen.

Weitere Details zur Lizenz entnehmen Sie bitte der Lizenzinformation auf http://creativecommons.org/licenses/by/4. 0/deed.de.

\section{Literatur}

1. ÖVE B 1:1976 (1976) Beeinflussung von Fernmeldeanlagen durch Wechselstromanlagen mit Nennspannungen über $1 \mathrm{kV}$.OVE, Wien

2. ÖVE/ÖNORM EN 50522 (2011) Erdung von Starkstromanlagen mit Nennwechselspannungen über $1 \mathrm{kV}$. OVE, Wien

3. IEC60479-1 (2018) 2018, Effects of current on human beings and livestock-Part 1: General aspects

4. ÖVE-EH 41 (1987) Erdungen in Wechselstromanlagen mit Nennspannungen über $1 \mathrm{kV}$. ÖVE, Wien

5. OllendorffF (1969) Erdströme. Birkhäuser, Basel

6. Srl S (2021) XGSLab TM UserGuide (Release 9.6.1-02/21), Bassano del Grappa, Italy

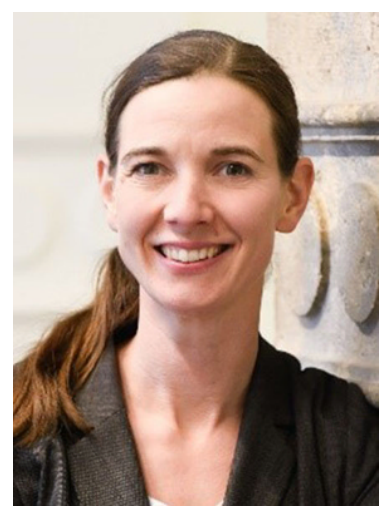

Katrin Friedl, studierte Elektrotechnik an der Technischen Universität Wien. Im Jahr 2013 promovierte sie an der Technischen Universität Graz im Bereich der elektromagnetischen Felder von Energieversorgungsanlagen. Danach arbeitete sie beim Übertragungsnetzbetreiber Austrian Power Grid AG im Anlagenmanagement mit den Schwerpunkten elektromagnetische Felder und Netzplanung. Seit 2019 ist sie am Institut für Elektrische Anlagen und Netze derTechnischen Universität Graz beschäftigt. Hier forscht und lehrt sie im Bereich der niederfrequenten magnetischen und elektrischen Felder, elektromagnetischen Beeinflussungen, sowie Erdung und Sicherheit von elektrischen Energiesystemen. 


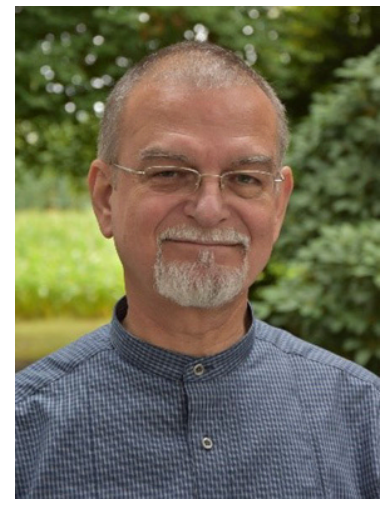

Reinhard Hirtler, geb. 1954, studierte ab 1973 Elektrotechnik mit dem Schwerpunkt Hochspannungstechnik und elektrische Anlagen an der Technischen Universität in Graz. Nach dem Studium war er ab 1983 bei der Verbundgesellschaft - dem heutigen Übertragungsnetzbetreiber Austrian Power Grid AG zuerst lange im Themenbereich der elektrischen Beeinflussung tätig und arbeitete danach bis 2001 im Bereich Netzplanung. Ab der Gründung der Gemeinnützigen Privatstiftung Elektroschutz durch Prof. Dr. Gottfried Biegelmeier im Jahre 1997 übernahm er für 20 Jahre die Aufgaben des Geschäftsführers der Stiftung. Seit 2010 ist er auch als Ingenieurkonsulent für Elektrotechnik aktiv. Daneben ergab sich eine umfangreiche Mitwirkungin verschiedenen nationalen und internationalen Normungsgremien insbesondere im Bereich der Errichtung von Hoch- und Niederspannungsanlagen, wobei die langjährige Mitarbeit bei IEC/TC 64/MT 4 zum Thema Wirkungen des elektrischen Stromes auf Menschen und Nutztiere besonders zu erwähnen ist.

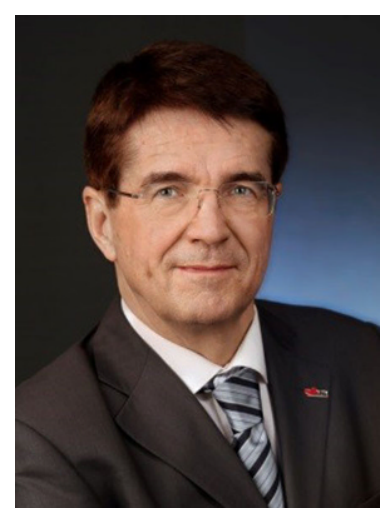

Lothar Fickert, promovierte 1974 an der Technischen Universität Wien und arbeitete danach 25 Jahre lang in der Industrie als Schutz- und Anlagentechniker in Netzplanung und Betrieb in der Energiewirtschaft (ELIN UNION, BROWNBOVERI, WIENSTROM). Seit 1998 wirkt er als Professor und Vorstand des Institutes für Elektrische Anlagen und Netze an der Technischen Universität Graz mit den Forschungsschwerpunkten elektrische Energiesysteme, Versorgungssicherheit und Schutztechnik. Er arbeitet in verschiedenen nationalen und internationalen Organisationen (IEC, CIGRE, CIRED, VDE, ÖVE usw.) mit und hat 9 Patente. Seit Oktober 2017 ist er Professor emeritus und widmet sich am Institut für Elektrische Anlagen und Netze neben seiner Kernprofession, der Schutztechnik, auch aktuellen Sternpunktfragen sowie modernen Lehr- und Lernmedien („Flipped Classroom“/100.000 Clicks). Seit 2018 ist er die Ombudsperson der Technischen Universität Graz.

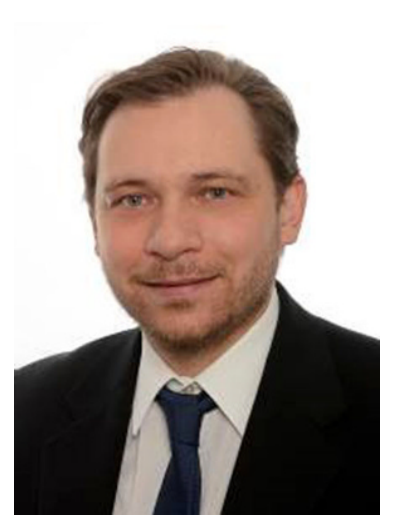

René Braunstein, schloss sein Studium der Elektrotechnik mit dem Schwerpunkt Elektrische Anlagen und Netze im Jahr 2007 mit Auszeichnung ab. Von 2007 bis 2012 war er Universitätsassistent am Institut für Elektrische Anlagen der TU Graz. Nach erfolgter Promotion wechselte er im Jahr 2012 zum steirischen Netzbetreiber - den Energienetzen Steiermark GmbH. In der Abteilung „Asset Management und Netztechnik Strom" beschäftigte er sich schwerpunktmäßig mit der Integration Erneuerbarer Energieträger ins Verteilernetz und den einhergehenden Ausbaukonzepten, dem Thema Spannungsqualität sowie der Betreuung von F\&E-Projekten und Abschlussarbeiten im Themenfeld der klassischen Anlagentechnik. In dieser Zeit war er als Mitglied des technischen Komitees für Beeinflussungsfragen an der Neuerstellung der TE30 beteiligt. Im Jahr 2021 wurde Herr Dr. Braunstein mit der Leitung der Abteilung „Mess- und Prüftechnik/Ladeinfrastruktur" betraut. Herr Braunstein ist Vertreter seines Unternehmens bei Österreichs Energie in den Themenfeldern Elektromobilität, Netze und (zukünftiger) Lasten, Isolieröle und Spannungsqualität. Seit 2020 ist er Vorsitzender des AK Spannungsqualität bei Österreichs Energie.

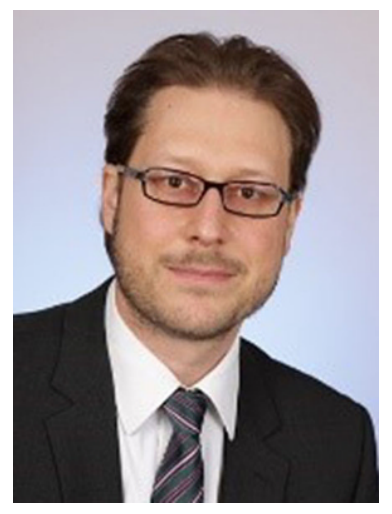

Robert Schürhuber, promovierte 2003 in Elektrotechnik an der Technischen Universität Wien. Von 2003 bis 2017 arbeitete er in verschiedenen Bereichen der elektrischen Energietechnik für die Firmen Siemens und Andritz Hydro, weiters war er als Lektor im Bereich der elektrischen Energietechnik an der Technischen Universität Wien tätig. Seit Oktober 2017 leitet er das Institut für Elektrische Anlagen und Netze an der Technischen Universität Graz 